\title{
Influence of exercise and vitamin D on the immune system against Covid-19: an integrative review of current literature
}

\author{
Matheus Ribeiro Bizuti ${ }^{1}$. Édina Starck ${ }^{1} \cdot$ Kimberly Kamila da Silva Fagundes $^{1}$. Josiano Guilherme Puhle ${ }^{2}$. \\ Lucas Medeiros Lima' ${ }^{1}$. Natan Rodrigues de Oliveira ${ }^{1}$. Guilherme Vinicio de Sousa Silva ${ }^{1}$. \\ Débora Tavares Resende e Silva ${ }^{3}$ (I)
}

Received: 25 August 2021 / Accepted: 23 February 2022 / Published online: 8 March 2022

(c) The Author(s), under exclusive licence to Springer Science+Business Media, LLC, part of Springer Nature 2022

\begin{abstract}
Respiratory infections of viral origin have become the leading cause of infectious diseases in the world. In 2020, the World Health Organization (WHO) declared a pandemic due to Severe Acute Respiratory Syndrome Coronavirus 2 (SARS-CoV-2), Coronavirus Disease 2019 (Covid-19). The pandemic caused by the new coronavirus has challenged the entire global health system, since Covid-19 has a high rate of morbidity and mortality. The immune response to the virus depends on factors such as age, genetics, nutritional status, physical status, as well as environmental factors. Despite scientific advances, so far, there is still no specific therapy for the disease. Thus, this study aims to analyze the contribution of physical exercise and maintenance and/or supplementation of vitamin $D$ to the strengthening of the immune system against viral infections, among them, Covid-19. Regular practice of moderate-intensity physical activity is responsible for promoting a reduction in the concentrations of pro-inflammatory cytokines (IL-6, TNF- $\alpha$ and IL-1 $\beta$ ), as well as triggering the increase in the production of anti-inflammatory cytokines (IL-4 and IL-10). In addition, hypovitaminosis D predisposes to the development of chronic diseases and infections. Therefore, in patients affected by Covid-19, the maintenance of vitamin D levels contributes significantly to the 0prevention of the cytokine storm. Thus, the association between maintaining vitamin D levels and performing moderate-intensity physical exercise is responsible for strengthening the immune system and, therefore, triggering a defense mechanism against infections by intracellular microorganisms, in which SARS -CoV-2.
\end{abstract}

Keywords Covid-19 $\cdot$ Vitamin D $\cdot$ Physical exercise $\cdot$ Immune system $\cdot$ Cytokine storm

Débora Tavares Resende e Silva

debora.silva@uffs.edu.br

Matheus Ribeiro Bizuti

matheus_ribeiro.bizuti@hotmail.com

Édina Starck

edina.starck@hotmail.com

Kimberly Kamila da Silva Fagundes

kimberlyfagundes08@gmail.com

Josiano Guilherme Puhle

puhlejosianoguilherme@gmail.com;

guilhermemeduffs@gmail.com

Lucas Medeiros Lima

lucasmedlima@gmail.com
Natan Rodrigues de Oliveira

natanr.oliveira@gmail.com.br

1 Department of Medicine, Federal University of Fronteira Sul, Chapecó, Santa Catarina, Brazil

2 The Graduate Program in Biomedical Sciences, Federal University of Fronteira Sul, Chapecó, Santa Catarina, Brazil

3 Department of Pathology. Graduate Program in Biomedical Sciences, Federal University of Fronteira Sul, Fronteira Sul, Highway SC 484 - Km 02, Chapecó, Santa Catarina 89815-899, Brazil 


\section{Introduction}

In December 2019, an outbreak of viral pneumonia was identified in the city of Wuhan, capital of Hubei province, China, whose etiologic agent was called Severe Acute Respiratory Syndrome Coronavirus 2 (SARS-CoV-2), responsible for the disease of the new coronavirus (Covid19). On March 11, 2020, the World Health Organization (WHO) declared a state of pandemic, due to Covid-19. In less than six months, the new coronavirus has hit virtually every country in the world, and many of these countries are already experiencing a second wave of the disease. Unlike other diseases associated with coronavirus, Covid19 is responsible for considerably higher rates of morbidity and mortality. This is due, to a certain extent, by the high rate of dissemination through asymptomatic or pre-asymptomatic carriers, who act as a reservoir of the disease $[1,2]$.

The main form of transmission is through contact with droplets containing viral particles, which can be eliminated by sneezing or coughing from infected people. The viral incubation period generally ranges from 2 to 14 days. Approximately $80 \%$ of individuals affected by the disease are asymptomatic or have mild symptoms, such as dry cough, fever, fatigue, dyspnoea and/or myalgia, which promotes rapid viral dissemination. The development of Covid-19 depends heavily on the interaction between the host's immune system and SARS-CoV-2, so that the immune response is influenced by factors such as nutritional status, age, sex and physical/metabolic status [2].

SARS-CoV-2 is highly pathogenic, being responsible, for this reason, for the high mortality rate. It is known that the coronavirus has a single stranded RNA as a genetic structure, which triggers diseases of both the gastrointestinal tract and the respiratory tract. Infection with the new coronavirus causes a series of immunological dysfunctions, providing an uncontrolled picture of cytokine secretion. In addition, SARS-CoV-2 is capable of infecting macrophages, monocytes, lymphocytes and dendritic cells, responsible for the cytokine storm. Individuals affected by Covid-19 release excessively cytokines, which are responsible, among other functions, to recruit leukocytes to different organs, among them, the lung, which thus causes the acute respiratory distress syndrome [2].

SARS-CoV-2 naturally mutates just like any other viruses, and although most of the genetic variations barely affect the way the virus behaves or threatens the population, some of them might change aspects like the spread and effectiveness of vaccines or medications (WHO). The arising issue of the new coronavirus variants promoted the creation of a denomination for the upcoming variants, that being the Variants of Interest (VOI), Variants of Concern
(VOC), and Variants of High Consequence (VOHC), though no VOHC has been detected yet. The WHO alongside partners like GISAID and Pango decided to name the new coronavirus variants as letters of the Greek alphabet, like Alpha, Beta, or Delta.

The Centers for Disease Control and Prevention (CDC) defines the VOI as a variant correlated with changes to receptor binding, neutralization by antibodies, decreased efficacy of medications, or potential increase in the disease's spread or severity. The variants B.1.525, or Eta as labeled by the WHO, B.1.526 (Iota), B.1.617.1 (Kappa), and C.37 (Lambda), are examples of the Variants of Interest.

It is known that the regular practice of physical activity is responsible for triggering significant improvements in the population's quality of life. With regard to the immune system, exercise, if practiced properly, can act to regulate the body's inflammatory conditions, as well as assist in immune defense mechanisms. In addition, the immune system is also influenced by the immunomodulatory role of vitamin D [1-6].

Vitamin D is responsible for regulating the mechanisms of calcium and phosphorus, playing a fundamental role not only in maintaining the mineralized skeleton, but also in the hormonal immunomodulatory process. Both metabolizing enzymes and vitamin D receptors are expressed by different cells of the immune system, including lymphocytes, monocytes, dendritic cells and macrophages. Furthermore, vitamin $\mathrm{D}$ has biological activity both in the innate immune system, through the secretion of antiviral peptides, and in the adaptive immune system [4-7].

Moreover, when talking about the effects of the vitamin D against the pathophysiological mechanisms of Covid-19, it is important to note the vitamin's ability to modulate proinflammatory and anti-inflammatory molecules [8] as well as control the cytokine storm that aggravates the disease [9, 10]. The vitamin $D$ also plays a role in damaging the protein envelope of the virus, stopping the genome from entering the cells [11-13].

That being said, it's already proposed that vitamin D supplementation could be related to a lower risk of respiratory tract infections [14], since studies have found. Also, studies have shown increased levels of the immune system's constituents that are related to the defense of respiratory viral infections [15]. Further, a study suggested that the association of regular physical exercises and vitamin D supplementation was related to a notable reduction of proinflammatory markers such as cytokines [16], an effect that could be useful in combating the cytokine storm from Covid- 19 .

The new coronavirus pandemic threatens the entire global health system, triggering an unprecedented situation of instability. So far, there are no specific therapeutic measures, the current treatment is done through symptomatic control of the disease. Studies show that vitamin D has antiviral effects, 
being able to prevent replication of the virus in question. In addition, vitamin $\mathrm{D}$, as well as regular physical activity, is effective in an anti-inflammatory and immunomodulatory way [3-7].

Therefore, this article aims to carry out an integrative review of the literature relating the role of physical exercise and vitamin D supplementation on the immune system in combating Covid-19. To this end, the bibliographic study included knowledge about the immunomodulatory and anti-inflammatory role of both vitamin D and physical exercise and, therefore, proposed the understanding of the most recent information on the pathological mechanisms of Covid-19 and its immunomodulatory manifestations, also comprising, its relationship with the physical conditions of individuals.

\section{Physical exercise and immune system}

Exercise is an essential factor in the release and control of cells, cytokines and interleukins from the immune system, and it is justified because it provides neurophysiological changes to the body, thus affecting all parts of the human body. Several studies have been conducted over the years with the aim of establishing relationships between exercise and the immune system, showing how exercise influences certain populations, which may or may not be affected by some specific disease [17-23].

It is well known that physical activity can affect the immune system, however, some factors must be taken into consideration when thinking about changes in this system. The type of exercise, its intensity, duration and periodicity, are the pillars that will favor certain situations within the immune system, causing various reactions and triggering varied responses [24].

In a study with the elderly population submitted to aerobic and resistance exercises it was observed a reduction of $32.7 \%, 31.8 \%, 32.1 \%, 21.9 \%, 33.7 \%$ and $24.3 \%$ in the mean values of Tumoral Necrosis Factor alpha (TNF- $\alpha$ ), Interleukin 6 (IL-6), CD3 count, CD4 count, CD8 count and CD4/ CD8 ratio, respectively. There was also a significant increase in immune system parameter values and a significant reduction in inflammation marker values after six months of aerobic training and moderate endurance; moreover, there were significant differences between the two types of physical training, where aerobic exercise achieved more remarkable effects [18].

University students were submitted to a protocol of 18 sessions of physical exercise lasting 50 min during the school period, using two groups with different types and intensities. The moderate continuous training group showed a decrease in the levels of TNF- $\alpha$ and pro-inflammatory cytokines. Although the high-intensity interval training decreased the depressive symptoms of the academics, it also increased the levels of TNF- $\alpha$ and IL- 6 in relation to the moderate continuous training group. This is justified by the higher level of physical stress evoked by the exercise protocol being more strenuous [19].

Long and/or high intensity exercises (above $80 \%$ VO2max and $2 \mathrm{~h}$ ) are associated with immunosuppression markers, in which there is an increase in the production of pro-inflammatory cytokines (IL-6, IL-8, TNF- $\alpha$ and IL-1) and a reduction in the immune system cells (NK cells, $\mathrm{T}$ and $\mathrm{B}$ lymphocytes and neutrophils). Thus, these individuals are more predisposed to be affected by infections (mainly upper respiratory tract infections), which may increase the risk of infection and worsening by Covid-19 [20, 25].

Short-term exercise (45/60 min) and moderate intensity (50/75\% VO2max) exercises performed at least three times a week positively affect the immune system, as increased leukocyte function, increased chemotaxis, degranulation, cytotoxic activity, phagocytosis and oxidative activity of neutrophils and macrophages and increased cytolytic activity of NK cells and NK cell activating lymphocytes [21]. Furthermore, there is a reduction in concentrations of pro-inflammatory cytokines (IL-6, TNF- $\alpha$ and IL-1 $\beta$ ), an increase in cytotoxic activity of NK and TCD $8+$ cells, and an increase in neutrophil function and B lymphocyte proliferation [22].

Moderate exercise associated with physical training (aerobic resistance training) favors an increase in macrophage phagocytosis, an increase in the percentage of TCD4 lymphocytes and a reduction in circulating TNF- $\alpha$ and IL- 6 . There is also an increase in the production of anti-inflammatory cytokines (IL-4 and IL-10) by T-cells [23].

In addition, physical exercise has a high potential in reducing communicable diseases, including viral pathologies. There is evidence that moderate-intensity exercise contributes to the reduction of upper airway infections, contributing to the reduction of symptoms and duration of infection, in order to provide a lower risk of mortality from infectious respiratory diseases. This is due to the detriment of the immunological modulation triggered by physical exercise, given that there is an increase in the anti-pathogenic activity of macrophages, while there is an increase in the circulation of cytokines and anti-inflammatory immunoglobulins. Thus, the viral load of the pathogen in the body, especially in organs such as the lung, is reduced. Furthermore, regular moderate-intensity physical exercise provides a series of beneficial changes to the body, which contributes to a better performance of the immune system, since it improves cardiorespiratory function, improves the vaccine immune response, improves lipid metabolism, glucose and insiluna, in short, a series of changes that favor the effects of antioxidant defense, contributing to the improvement of 
immunological surveillance, reducing the systemic inflammatory Picture $[3,18]$.

Recently published evidence shows that a subgroup of patients with severe cases of the new coronavirus infection has a cytokine storm syndrome (hypercitokineemia) triggering a high inflammatory response. The triggering factor of this process is hemophagocytic lymphistiocytosis (LHH), characterized by immune hyperactivation that occurs when NK cells and cytotoxic T lymphocytes do not eliminate activated macrophages, leading to excessive production of proinflammatory cytokines [26].

The main symptoms of LHH include: continuous fever, cytopenias, hyperferritinemia and pulmonary involvement. A cytokine profile similar to LHH is associated with the most severe phenotype of the disease caused by Covid19 , characterized by increased IL-2 and IL-7, granulocyte colonies, IFN- $\gamma$, macrophage inflammatory protein $1-\alpha$ and TNF- $\alpha$ [27].

Regular practice of moderate-intensity physical exercise helps neutralize inflammatory processes. In animal models, exercise provided a significant decrease in hippocampal microglia, as well as an increase in anti-inflammatory cytokines, such as IL-6, IL-1ra and IL-10. Furthermore, there was a reduction in the production of TNF- $\alpha[28,29]$.

IL-6, classically classified as a pro-inflammatory cytokine, starts to play an anti-inflammatory role as a result of IL-6-induced acute phase proteins, which have an antiinflammatory effect when originating from skeletal muscles. The production of IL-6 is determined by the stimulation of $\mathrm{Ca}^{2+}$ and glycogen in association with muscle contraction. Generally, the IL-6 peak occurs moments after the end of physical activity, in order to return to baseline levels hours later. In moderate intensity activities, there is a modest increase in this cytokine, with no peak in IL-6 concentration, which causes this anti-inflammatory mediator to remain at constant levels in the body, in order to exert control over the immune system and, on the other hand, improve defenses against infectious processes [30, 31].

In addition to this factor, moderate-intensity exercise provides a decrease in pro-inflammatory mediators, especially IL-18, C-reactive protein, TNF- $\alpha$ and IL- $1 \beta$ and an exacerbated increase in IL-10. The high levels of anti-inflammatory IL-10 is able to neutralize the effects of pro-inflammatory cytokines and prevent the infiltration of these cells into the tissues, in order to decrease the levels of adhesion molecules such as ICAM-1 [28]. Several mechanisms are involved in the anti-inflammatory effects provided by physical exercise, including: reduction of visceral fat; increased production of cortisol, adrenaline, prolactin, growth hormone, among others, in order to influence the flow of leukocytes; decreased expression of TLR in immune cells; and increased levels of anti-inflammatory myokines influenced by skeletal muscle $[32,33]$.
The Covid-19 pandemic has become a clinical threat to the world, and it is consensus that the way to reduce the rate of contamination and spread is through social withdrawal. However, the practice of moderate intensity exercises at home is recommended. Moderate exercise-induced immunomodulation can be an important tool for improving immune responses against the progression of SARS-CoV-2 infection [25].

\section{Vitamin D and immune system}

A variety of studies approach the inverse association between vitamin $\mathrm{D}$ and the occurrence of infection $[34,35]$. It is known that vitamin D and its pro-hormones are able to interact and modulate the immune system's activities, in view of the wide expression of its receptors in the tissues of the organism, including the immunitary cells. In this sense, vitamin D seems to act in regulating the differentiation of lymphocytes, macrophages and NK cells, besides influencing the production of autoantibodies and cytokines, being able to modulate the levels of pro-inflammatory and antiinflammatory molecules [8].

Hypovitaminosis D seems to be related to an increased risk of developing chronic diseases and susceptibility to infections [8]. In contrast, studies have concluded that the correction of vitamin D deficiency was responsible for a reduction of up to $12 \%$ in respiratory infections, while in the most severe cases of deficiency it reduced its occurrence by $70 \%$ [36]. Faced with infection by the new coronavirus, a recent study with 176 elderly patients in an ICU identified the occurrence of hypovitaminosis $\mathrm{D}$ that varied according to the reference values of 1,25-dihydroxyvitamin $\mathrm{D}(1,25$ $(\mathrm{OH}) 2 \mathrm{D}$ ), in which levels below $30 \mathrm{ng} / \mathrm{mL}$ were found in 93.8\% of hospitalized patients [37]. Other studies observed a statistically significant relationship $(p=0.046)$ between mortality from Covid-19 and 25(OH)D concentration, as well as low plasma levels in patients with positive tests [38, 39].

A member of the steroidal hormone family, vitamin $\mathrm{D}$ has a nuclear-type receptor. Vitamin D receptors (VDR) are present in several immunoreactive cells, such as macrophages, neutrophils and epithelial cells, and are activated by contact with viral and bacterial ligands [5]. After its coupling to specific receptors it is converted to 25 -hydroxyvitamin D $(25(\mathrm{OH}) \mathrm{D})$ and $1,25(\mathrm{OH}) 2 \mathrm{D}$. The $25(\mathrm{OH}) \mathrm{D}$, which defines the vitamin $\mathrm{D}$ status and has autocrine activity, participates in the production of unclivated cathelicidin, which is subsequently cleaved and acquires the formation of active cathelicidin (LL37), that has cytotoxic action [7, 34, 35].

Similarly to innate immunity, adaptive immunity is also regulated by $1,25(\mathrm{OH}) 2 \mathrm{D}$, directly modulating the activation of $\mathrm{T}$ cells and antigen presenting-cells [34]. Toll-like receptor stimulation by TLR2/IL of macrophages positively 
regulates vitamin $\mathrm{D}$ receptor, expressed in different cell types, and the 25-hydroxyvitamin D-1-alpha-hydroxylate, converting $25(\mathrm{OH}) \mathrm{D}$ to $1,25(\mathrm{OH}) 2 \mathrm{D}$ and the expression of cathelicidin mRNA [40].

The 1,25 D signaling, in turn, suppresses the transcription of genes that codify pro-inflammatory cytokines from $\mathrm{T}$ helper 1 (Th1), interferon- $\gamma($ IFN- $\gamma)$ and interleukins IL-17 and IL-21, in addition to enabling treated $\mathrm{T}$ cells to obtain regulatory $\mathrm{T}$ cell (Treg) characteristics, thus ensuring the suppression of the pro-inflammatory state $[5,7,35]$. Some studies mention the suppression of inflammatory cytokines and chemokines as a possible reducer of the cytokine storm observed in patients with Covid-19 [5].

Considering the knowledge about the performance of SARS-CoV-2 by converting angiotensin enzyme 2 (ACE 2), it was observed a potential correlation between 1,25 $(\mathrm{OH}) 2 \mathrm{D}$ and the modulation of ACE expression in the lungs [41]. Therefore, once again, vitamin $\mathrm{D}$ can prevent cytokine storms in patients affected by the new coronavírus [42].

Furthermore, there is a recent approach regarding the presence of partially hydrophobic proteins, contained in the SARS-CoV-2 virus, which initiate the non-bending protein (UPR) response due to its contact with the dense environment, preventing the occurrence of metabolic processes and triggering a response to metabolic stress. The presence of UPR activates the NF- $\kappa B$ pathway leading to a hyperexpression of inflammatory gene promoters, thus promoting viral replication and systemic inflammation [43].

In this sense, through its products, vitamin $\mathrm{D}$ inhibits the activation of NF- $\kappa \mathrm{B}$, featuring the profile as anti-inflammatory [43]. Therefore, it is considered that vitamin $\mathrm{D}$ actuates in different components of the immune system, namely: neutrophils, dendritic cells, macrophages, $\mathrm{B}$ and $\mathrm{T}$ cells, antibodies and cytokines (Table 1) [34, 44, 45].

\section{Neutrophils}

In the context of severe infections, there is an increase in the presence of neutrophils. Because these cells are the main sources of cathelicidin, infectious control is made possible [45].

\section{Dendritic cells (DC)}

DC are target of the immunomodulatory effects of vitamin $\mathrm{D}$, acting by immunogenic or tolerogenic signals [45]. Immature DC has lower levels of MHC class II and co-stimulatory molecules-such as CD40, CD80 and CD86-which results in decrease in the antigen presentation and secretion of IL-12, while there is an increase in IL-10 [45, 46]. In vitro inhibition of monocyte differentiation into $\mathrm{CD}$ was also observed [8].

\section{Monocytes and Macrophages}

Monocyte proliferation is amplified in the presence of 1,25 $(\mathrm{OH}) 2 \mathrm{D}$. In addition, vitamin D promotes the expression of the antimicrobial peptide cathelicidin, improving the bactericidal capacity of macrophages and benefiting innate immunity. Macrophages, as well as monocytes, constitutively express VDR and, in these cells, vitamin D stimulates its own actions, as it expands the receptor's expression. [44]

\section{T and B cells}

Studies approach the expression of $\mathrm{T}$ and $\mathrm{B}$ cells activating enzymes and of nuclear VDR as a potential effect of vitamin $\mathrm{D}$ in the cells of the adaptive immune system, modifying directly the cellular proliferation and the cytokines profile $[8,45,46]$. Vitamin D has inhibitory effect in production of $\mathrm{T}$ lymphocytes and cytokines secretion, in a similar manner, the CD8 T cell has its proliferation supressed due to treatment with $1,25(\mathrm{OH}) 2 \mathrm{D}$ and its cytotoxic activity attenuated by the vitamin $[8,44,47]$.

It is known that the active metabolite of vitamin $\mathrm{D}-1,25$ (OH) 2D-inhibits the lymphocyte T helper 1 (Th1), which leads to an increased T helper 2 (Th2) response [44]. Thus, an increase in the number and also the function of Treg lymphocytes may occur [8].

\section{Antibodies}

There are reports of inhibition of the proliferation of B cells, as well as their differentiation into antibody secreting cells, due to the presence of 1,25 (OH) 2D [44]. As a consequence, a suppression of the release of antibodies, such as immunoglobulin $\mathrm{E}$ (IgE) and $\mathrm{G}(\mathrm{IgG})$, by B lymphocytes is observed $[8,44]$.

\section{Cytokines:}

Due to the stimulation of Th2 cells by the action of vitamin $\mathrm{D}$, an increase in the production of IL-4, IL-5, IL-10 and IL-13 is observed, molecules with anti-inflammatory properties. In addition, due to Th1 suppression, there is a decline in IL-2, IL-6, IL-12, IFN- $\gamma$ and TNF- $\beta$ levels, cytokines that promote pro-inflammatory effects $[8,42]$.

The inhibition of IL-17 secretion by T helper 17 (Th17) cells and the induction of Treg and IL-10 cell development by $1,25(\mathrm{OH}) 2 \mathrm{D}$ was approached in a study [47]. Vitamin 
Table 1 Relationship of vitamin D and physical exercise with immune system cells and their mechanisms of action

\begin{tabular}{lll}
\hline Cells & Signaling Mechanism & Action \\
\hline Neutrophils & VDR and Cathelicidin & $\begin{array}{l}\text { Vitamin D: Increase in the presence of Neutrophils, and in } \\
\text { infectious control } \\
\text { Exercise: Long and/or high intensity exercises decreased the } \\
\text { presence of Neutrophils. Short-term exercise (45/60 min) }\end{array}$ \\
& $\begin{array}{l}\text { and moderate intensity (50/75\% VO2max) exercises } \\
\text { performed at least three times a week positively affect the } \\
\text { immune system, as increased activity of neutrophils }\end{array}$
\end{tabular}

Dendritic Cells (DC) Immunogenic or Tolerogenic signals, CD40, CD80, CD86, IL-10, IL-12 and MHC class II

Monocytes and Macrophages $1,25(\mathrm{OH}) 2 \mathrm{D}$, VDR, and Cathelicidin
Vitamin D: Decrease in antigen presentation and secretion of IL-12, and increase in secretion of IL-10. Inhibition of monocyte differentiation into DC

Vitamin D: Monocyte proliferation is amplified. Promotes the expression of Cathelicidin, improving bacterial capacity of macrophages and benefiting innate immunity. Expands VDR's expression

Exercise: Short-term exercise (45/60 $\mathrm{min}$ ) and moderate intensity (50/75\% VO2max) exercises performed at least three times a week positively affect the immune system, as increased activity of macrophages. Moderate exercise associated with physical training (aerobic resistance training) favors an increase in macrophage phagocytosis

T cells

1,25(OH)2D, VDR, IL-1, IL-2, IL-4, Vitamin D: Inhibitory effect in production of T lymphocytes IL-5, IL-6, IL-8, IL-10, IL-12, IL-13, IFN- $\gamma$, TNF- $\alpha$ and TNF- $\beta$ and cytokines secretion. Supression oof CD8 T cells proliferation, and its cytotoxic activity attenuated by the vitamin. Inhibition of Th1 and increased Th2 response. Increase in number and function of Treg lymphocytes. Increase in the production of IL-4, IL-5, IL-10 and IL-13, with anti-inflammatory effects. Decline in IL-2, IL-6, IL-12, IFN- $\gamma$ and TNF- $\beta$ levels, with pro-inflammatory effects

Exercise: Aerobic and resistance exercises caused a reduction in the mean values of TNF- $\alpha$, IL- 6 , CD3 count, CD4 count, CD8 count and CD4/CD8 ratio. Long and/or high intensity exercises (above $80 \% \mathrm{VO} 2 \mathrm{max}$ and $2 \mathrm{~h}$ ) are associated with an increase in the production of pro-inflammatory cytokines (IL-6, IL-8, TNF- $\alpha$ and IL-1) and a reduction in T cells. Short-term exercise (45/60 min) and moderate intensity (50/75\% VO2max) exercises performed at least three times a week caused a reduction in concentrations of pro-inflammatory cytokines (IL-6, TNF- $\alpha$ and IL-1 $\beta$ ), an increase in cytotoxic activity TCD $8+$ cells. Moderate exercise associated with physical training (aerobic resistance training) favors an increase in the percentage of TCD4 lymphocytes and a reduction in circulating TNF- $\alpha$ and IL-6. There is also an increase in the production of anti-inflammatory cytokines (IL-4 and IL-10) by T-cells

B cells $1,25(\mathrm{OH}) 2 \mathrm{D}, \mathrm{VDR}, \mathrm{IgE}$ and IgG

Vitamin D: Inhibition of proliferation of B cells, and its differentiation into antibody secreting cells. Consequently, the release of antibodies is suppressed, such as $\mathrm{IgE}$ and $\mathrm{IgG}$

Exercise: Long and/or high intensity exercises (above $80 \%$ $\mathrm{VO} 2 \mathrm{max}$ and $2 \mathrm{~h}$ ) are associated with a reduction in B cells. Short-term exercise (45/60 min) and moderate intensity (50/75\% VO2max) exercises performed at least three times a week caused an increase in B cells proliferation reducing the levels of this interleukin. Finally, the reduction of the IL-12 synthesis due to the vitamin D results in a modification of the Th1 and Th2 axis [46]. 


\section{Imunne system and clotting disorders}

After the initial activation of the immune system, in the face of SARS-CoV-2 infection, there is also activation of the clotting pathway, the so-called thrombo-inflammation state. Although its pathogenesis is not fully understood, the hypercoagubility state developed in its pathogenesis has been increasingly addressed and observed [48].

The expression of the human angiotensin-converting enzyme 2 (ACE2) receptor, the binding site of the spike protein (S protein) of the SARS-CoV-2 virus, can be visualized beyond lung cells as in blood vessels, brain and heart. The deregulated renin-angiotensin-aldosterone system (RAAS) is affected by the virus, decreasing its converting activity. Thus, there is no conversion of angiotensin II and, consequently, its active pro-inflammatory and prothrombotic effects, such as the generation of reactive oxygen species mediated by NADPH oxidases [49].

The increase in tissue factor, influenced mainly by IL-6 and also by the complement factor C5a, leads to vessel wall disruption, triggering the production of thrombin, fibrinogen and fibrin. Among the different fronts of action, thrombin and coagulation factor $\mathrm{X}$, in addition to activating fibrin and fibrinogen, stimulate the release of more inflammatory cytokines, exacerbating the immune response. Thus, levels of IL-6, D-dimer and fibrinogen confirm the event of thromboinflammation in Covid-19, leading not only to microvascular thrombosis, but also to multiple organ dysfunction syndrome and death [48-50].

In the face of the innate immune response, extracellular neutrophil traps (NETs) are released to prevent the spread of microorganisms. In the exacerbation of the immune response triggered by SARS-CoV-2 infection, together with NETS we have the presence of prothrombotic molecules, tissue factor, factor XII and fibrinogen, which induce thrombogenesis. In addition to these, other pathways that stimulate thrombogenesis are being investigated as contributors to this process [48-50].

\section{Vitamin D and Covid-19}

Vitamin D is obtained through diet, sunlight and supplementation. There are two main types of vitamin D: vitamin D2, found in mushrooms exposed to ultraviolet radiation; and vitamin D3, found mainly in fish with a high amount of oil [51]. Intake of vitamin D, both D2 and D3, takes this micronutrient to the liver, where a hydroxylation reaction occurs by the enzyme vitamin-D-25-hydroxylase (CYP2R1) to 25-hydroxyvitamin D (25 (OH) D). Subsequently, in the kidneys, $25(\mathrm{OH}) \mathrm{D}$ is metabolized by the enzyme 25 -hydroxyvitamin D-1 $\alpha$-hydroxylase (CYP27B1) to its active form, 1,25-dihydroxyvitamin $\mathrm{D}(1,25(\mathrm{OH}) 2 \mathrm{D})$. The 1,25 (OH) $2 \mathrm{D}$ then binds to intracellular receptors called vitamin $\mathrm{D}$ receptor (VDR), influencing gene transcription and exerting its physiological functions (Fig. 1) [7].

The 1,25(OH)2D acts on phosphate and calcium homeostasis. In the intestine, its function is to promote the absorption of intestinal calcium, passively stimulating bone mineralization. In the kidneys, its function is to promote calcium reabsorption by the renal tubules, which helps to maintain an adequate calcium-phosphate product that crystallizes in the collagen matrix. Regarding the effects on bone, 1,25
Fig. 1 Vitamin D metabolization mechanism. Vitamin D is obtained through food, the action of ultraviolet light and exogenous supplementation. In the liver, it undergoes the action of the enzyme CYP2R1 (25-hydroxylase), being converted into $25(\mathrm{OH}) \mathrm{D}$. When this molecule reaches the kidneys, it is converted by CYP27B1 (1-alpha-hydroxylase) to $1,25(\mathrm{OH}) 2 \mathrm{D}$, the active form of the vitamin. Source The Authors

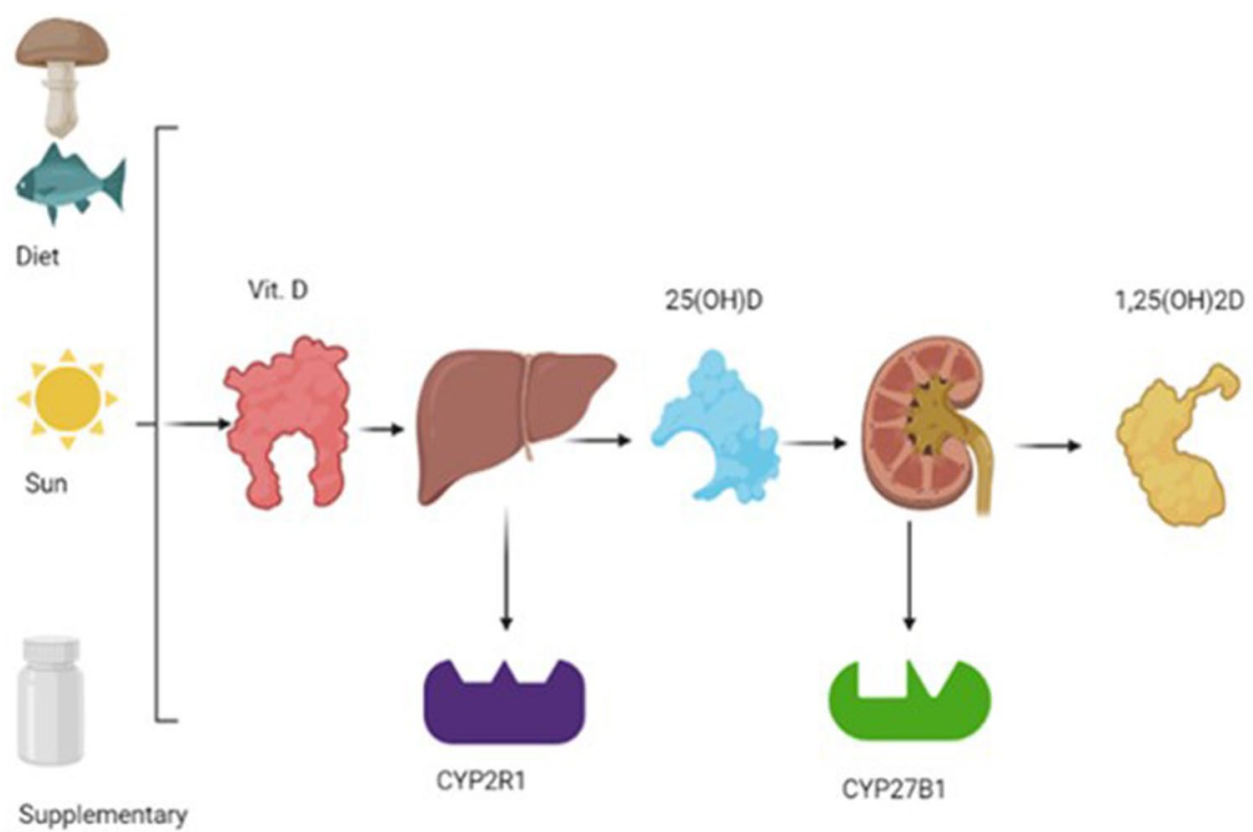


$(\mathrm{OH}) 2 \mathrm{D}$ is able to induce the expression of osteocalcin and stimulate the activator of nuclear factor kappa-B receptor (RANK) - dependent bone resorption. Regarding the endocrine system, 1,25 $(\mathrm{OH}) 2 \mathrm{D}$ acts on the negative feedback of parathyroid hormone (PTH), inhibiting its production and leading to a decrease in bone resorption and a consequent increase in urinary calcium excretion. Furthermore, it also induces the production of fibroblast growth factor 23 (FGF23), increasing urinary phosphate excretion [7].

In the disease caused by the new Coronavirus, the inflammatory process plays an important role in the evolution and control of pathophysiological mechanisms, especially with regard to the cytokine storm, as it is related to the worsening of the disease $[9,10]$. In infections, the inflammatory process is extremely important, however, when it occurs decompensated, it can lead to tissue damage and, in the case of Covid-19, it mainly affects the lungs, and may also occur in other organs [52]. Cytokine storms occur when control of pro-inflammatory cytokine and chemokine release pathways by the innate immune system is lost, resulting in exacerbated activation of the adaptive immune system [53].

In viral infections, vitamin D plays a suppressive role in the immune system. This is due to its anti-inflammatory role, suppressing the proliferation of CD8 T cells and expression of several cytokines with a pro-inflammatory role, such as IL-12, which are important players in the early stages of viral infection. This anti-inflammatory action is also related to the ability of vitamin $\mathrm{D}$ to prevent the cytokine storm in patients with SARS-CoV-2 [42, 54].

Vitamin D, in turn, acts on the immune system by regulating the action of macrophages, monocytes and antigenpresenting cells [7]. It also plays a direct role through VDRRXR (Vitamin D and Retinoid X Receptors, respectively) signaling, which then promotes the production of LL-37, an endogenous antimicrobial cathelicidin that acts by destabilizing the membrane of bacteria and fungi that invade the body $[40,45,55,56]$. In the case of viruses, vitamin $\mathrm{D}$ acts more directly by compromising the protein envelope and preventing the entry of genetic material into cells [57-59]. Allied to this, vitamin D is also responsible for suppressing the production of pro-inflammatory cytokines, such as IL-2, IL-6 and IL-17 [55, 60, 61].

Despite its considerable role in the immune system, the additional intake of vitamin $\mathrm{D}$ has not been proven to be beneficial to those who already have an established picture of disease caused by SARS-CoV-2 [62, 63]. Furthermore, no improvement has been identified in critically ill patients with vitamin D deficiency after ingestion of high doses either [64]. The only correlation that could be identified is that of better prognosis for patients with vitamin D deficiency who took high doses before contracting the disease or in early stages, due to better modulation of the immune system and prevention of cytokine storm, thus avoiding further multiplication of SARS-CoV-2 and potential damage caused by the cytokine storm [67-69].

Sengupta et al. (2021) cite the protective role of vitamin $\mathrm{D}$ in restricting the production of inflammatory cytokines and proliferation of pro-inflammatory cells, necessary for the development of the cytokine storm and, consequently, the thromboimmune effects caused by SARS-CoV-2. Vitamin D metabolites regulate pro and antithrombotic events of the coagulation cascade, reducing TNF-alpha-induced expression and tumor factor activity, in addition to upregulating the expression of the factor pathway inhibitor [48].

Studies demonstrate the regulation of RAAS by $1,25(\mathrm{OH}) 2 \mathrm{D}$, inhibiting the expression of ACE and, consequently, the entry of SARS-CoV-2. In addition, there are effects that prevent endothelial cell dysfunction and vascular thrombosis, occurring due to stabilization of the vascular endothelial membrane, through a non-genomic pathway [50, 57]. Uberti et al. (2014), through their in vitro study with human umbilical vein cells, proved the action of vitamin D in the prevention of apoptosis and autophagy, through superoxide anions and nitric oxide production [58].

\section{Exercise, vitamin D and immune system}

In a review discussing dietary supplementation in athletes, it is suggested that vitamin D supplementation may be associated with a reduced risk of upper respiratory tract infections in both physically active populations and the general population [14].

At the end of a 14-week vitamin D replacement protocol in athletes, studies observed an increase in secretory immunoglobulin A (SIgA) and cathelicidin, components of the immune system used to defend oral and respiratory viral infections [15].

On the other hand, in a supplementation protocol of 3,000 IU vitamin D for 12 weeks in a population of soccer athletes during the winter, studies [59] did not observe effects on the participants' immune profile. A possible cause would be that the immunological components evaluated were already, at the beginning of the study, at their normal or similar intervals to the general population.

In a study evaluating cytokines and other inflammatory markers before and $12 \mathrm{~h}$ after running a $100 \mathrm{~km}$ run in amateur ultra-marathon runners, the researchers [16] applied a vitamin D supplementation protocol to part of the study participants. Thus, it was observed that the daily use of 10,000 IU of vitamin D for 2 weeks generated a significant reduction in circulating levels of IL- $1 \beta$ and an increase in circulating TNF- $\alpha$ and miR-155 after the $100 \mathrm{~km}$ course compared to pre-collection running, whereas in the group without supplementation no such trend was observed (Fig. 2). 
Fig. 2 Performance of vitamin $\mathrm{D}$ and physical exercise in the immune system. Vitamin D supplementation associated with physical exercise contributes to the reduction of the risk of upper respiratory tract infection, given that there is an increase in the levels of SIgA, TNF- $\alpha$ and catelecidines. On the other hand, there is a significant reduction in circulating levels of the pro-inflammatory interleukins IL-6 and IL-1 $\beta$. Source The Authors

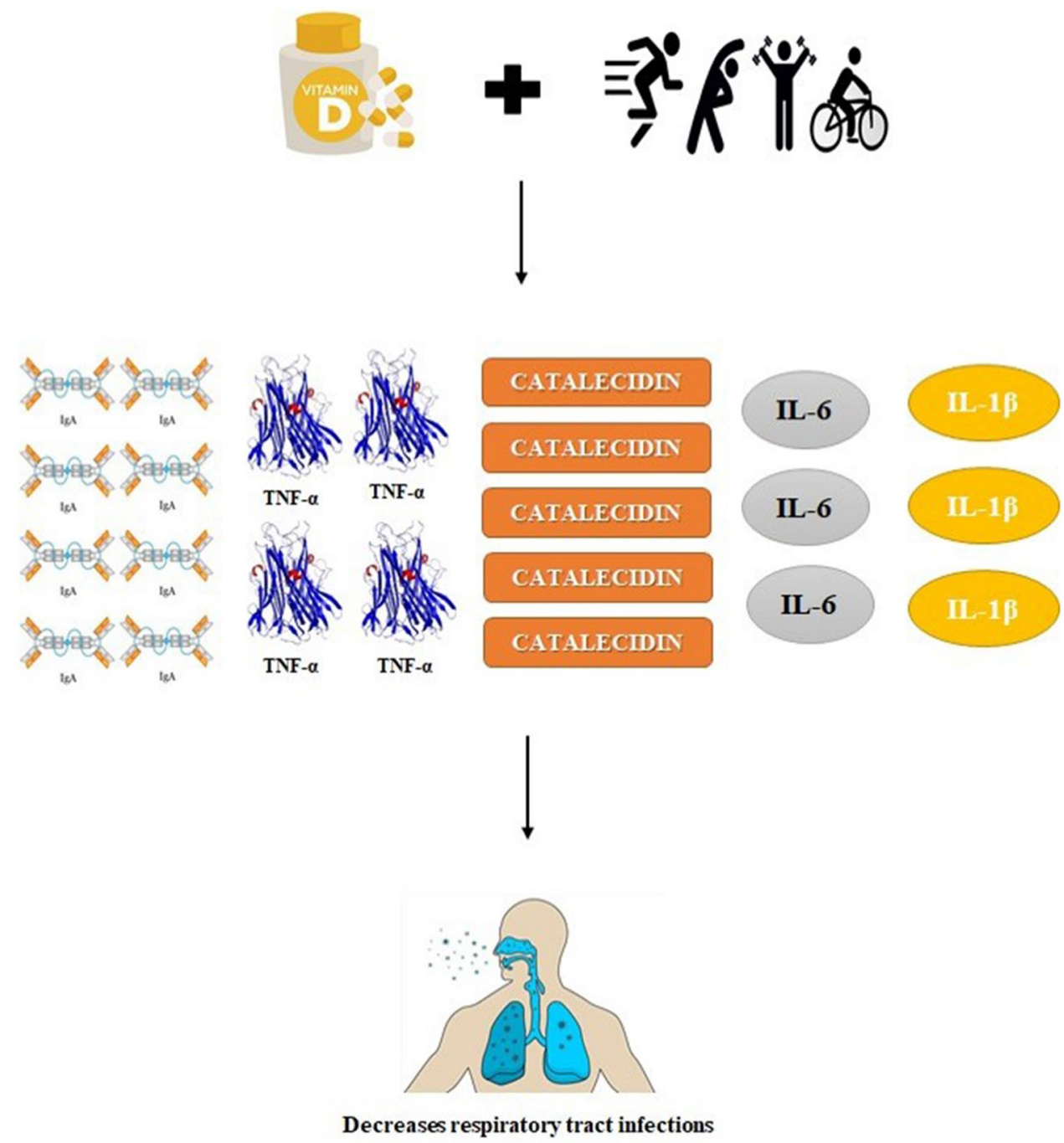

Decreases respiratory tract infections
Associating inflammatory indices with physically active and non-physically active university women and vitamin D levels above or below the ideal value, researchers [65] found that regular exercise was associated with higher serum levels of $25(\mathrm{OH}) \mathrm{D}$, a better composition profile reduction of the pro-inflammatory cytokine IL-6.

Recently, a review [66] using 14 observational studies on serum 25-hydroxyvitamin D levels suggests that vitamin D concentrations are inversely associated with the incidence and severity of Covid-19, and replacement may be a suggested measure at the individual level, but at the health level Public health depends on observational studies. The mechanisms by which vitamin $\mathrm{D}$ would reduce the risk and severity of Covid-19 infection would be by maintaining the epithelial layer, reducing survival and replicating the virus by inducing the production of cathelicidins and defensins, as well as reducing inflammation and production of pro-inflammatory cytokines, reducing the risk of the "cytokine storm" and increasing concentrations of the angiotensin-converting enzyme 2, which reduces the availability of Angiotensin 2 and the damage caused by SARS-CoV-2 (Fig. 3) (Fig. 4) $[67,68]$.

In a literature review that evaluated the practice of exercise, nutrition and the use of medications during the Covid19 pandemic in elderly populations, the authors highlight the importance of physical activity and an adequate nutritional profile for positive outcomes in Covid-19, and suggest that studies evaluating vitamin D supplementation in the context of the disease should be carried out to confirm the real benefit in patients at high risk for the disease, since studies at the moment evaluated vitamin D deficiency more in the development of symptoms [68].

\section{Conclusion}

Vitamin D is responsible for playing an essential role in maintaining phosphate, calcium and bone metabolism. When 1,25 $(\mathrm{OH}) 2 \mathrm{D}$ is produced, it is responsible for acting in a paracrine and autocrine manner, in order to carry 


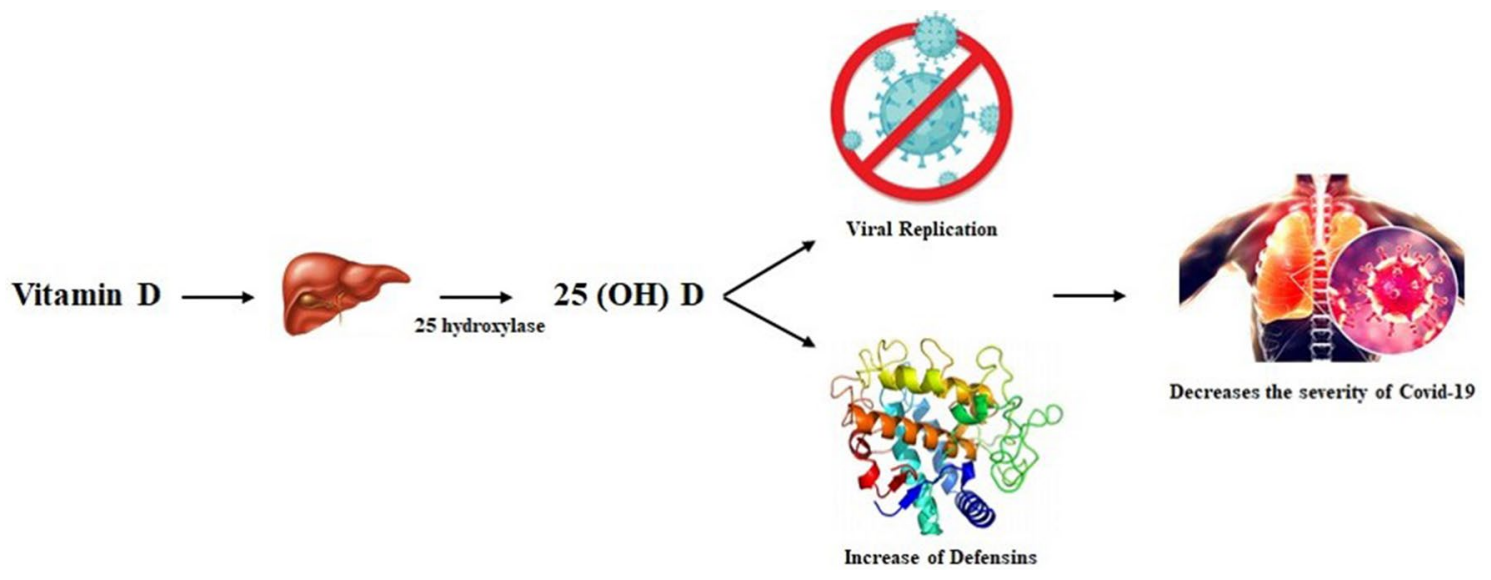

Fig. 3 Role of vitamin D in the control of Covid-19. Vitamin D is converted to $25(\mathrm{OH}) \mathrm{D}$ by the liver by the action of the enzyme 25 hydroxylase. Thus, the epithelial layer is maintained in order to provide, through the action of cathelicidins and defensins, a reduction in survival and viral replication. In patients affected by Covid-19, there is a lower incidence of severe forms of the disease in individuals who used vitamin D supplementation.. Source The Authors

\section{COVID-19 \\ Immune hyperactivation \\ $\mathrm{LHH}$ \\ Thrombo-inflammation}

\section{PHYSICAL EXERCISE MODERATE}

Pro-inflammatory cytokines (TNF-a / IL-6)

if Anti-inflammatory cytokines (IL-4 / IL-10)

仓 Leukocytes function

Macrophage phagocytosis

i TCD4 lymphocytes

\section{HIGH-INTENSITY}

Pro-inflammatory cytokines (TNF- $\alpha$ / IL-6 / IL-8 / IL-1)

$\checkmark$ Immune system cells (NK cells, T and B lymphocytes, neutrophils)
个 Cathelicidin (antimicrobial activity)

î Treg

Anti-inflammatory profile (IL-4 / IL-5 / IL-10 / IL-13)

Pro-inflammatory cytokines (IL-2 / IL-6 / IL-12 / IFN-y / TNF- $\beta$ )

Fig. 4 Schematic representation of immunological changes triggered by physical exercise and vitamin D. Source The Authors

out the modulation of the innate and adaptive immune system, acting simultaneously in both the stimulation of the innate immune system, for by suppressing the production of cytokines, in order to reduce the burden of pathogens, as well as in the adaptive immune system, by decreasing its functioning, that is, of hyperactivation, in the face of an infection, allowing the organism an adequate response to pathogen load.

In addition, 1,25 (OH) 2D performs antiviral activities, as well as acting on the inflammatory response to viral infection by releasing cathelicidin, suppressing overexpression of pro-inflammatory cytokines and modulating toll-like receptors and NK cells. The 1,25 (OH) 2D, in addition to exerting its antiviral and immunomodulatory effects, also acts as a regulator of the renin-angiotensin pathway, in order to negatively conduct the expression of the angiotensin-2 converting enzyme, which acts as a receptor cell responsible for SARSCoV-2 infection.

Therefore, maintaining vitamin $\mathrm{D}$ levels between 40 and $60 \mathrm{ng} / \mathrm{mL}(100-150 \mathrm{nmol} / \mathrm{L})$ is able to minimize the risk and severity of Covid-19 infection. Associated with this, the performance of physical activity on a regular and moderate basis, is able to promote a framework of protection against infections triggered by intracellular microorganisms, since exercise provides an increase in the body's antioxidant activity, thus providing an increased immune surveillance. 
Author contributions MRB and DTRS contributed to the conception, project administration and supervision. ES, KKSF and JGP, contributed to the formal analysis, investigation, and methodology. DTRS supervised the methodology. LML, NRO, and GVSS contributed to the writing and translation of the original draft. MRB and DTRS revised the final work.

Funding Not applicable.

Data availability Not applicable.

Code availability Not applicable.

\section{Declarations}

Conflict of interest The authors declare that there are no competing conflicts of interest.

Ethical approval Not applicable.

Consent to participate Not applicable.

Consent for publication Not applicable.

\section{References}

1. Dhama K, Khan S, Tiwari R et al (2020) Coronavirus disease 2019-COVID-19. Clin Microbiol Rev 33:e00028-e120. https:// doi.org/10.1128/cmr.00028-20

2. Machhi J, Herskovitz J, Senan AM et al (2020) The natural history, pathobiology, and clinical manifestations of SARS-CoV-2 infections. J Neuroimmune Pharmacol 21:1-28. https://doi.org/ 10.1007/s11481-020-09944-5

3. da Silveira MP, Fagundes KKS, Bizuti MR et al (2020) Physical exercise as a tool to help the immune system against covid19: an integrative review of the current literature. Clin Exp Med 21:15-28. https://doi.org/10.1007/s10238-020-00650-3

4. Muralidar S, Ambi SV, Sekaran S et al (2020) The emergence of COVID-19 as a global pandemic: understanding the epidemiology, immune response and potential therapeutic targets of SARSCoV-2. Biochimie 179:85-100. https://doi.org/10.1016/j.biochi. 2020.09.018

5. Chandran M, Maung AC, Mithal A et al (2020) Vitamin D in COVID - 19: Dousing the fire or averting the storm?-A perspective from the Asia-Pacific. Osteoporos Sarcopenia 6:97-105. https://doi.org/10.1016/j.afos.2020.07.003

6. Ali N (2020) Role of vitamin D in preventing of COVID-19 infection, progression and severity. J Infect Public Health 13:13731380. https://doi.org/10.1016/j.jiph.2020.06.021

7. Charoenngam N, Holick MF (2020) Immunologic effects of vitamin D on human health and disease. Nutrients 12:2097. https:// doi.org/10.3390/nu12072097

8. Marques CDL, Dantas AT, Fragoso TS et al (2010) The importance of vitamin D levels in autoimmune diseases. Braz J Rheumatol 50:67-80. https://doi.org/10.1590/S0482-5004201000 0100007

9. Yang P-H, Ding Y-B, Xu Z et al (2020) Increased circulating level of interleukin- 6 and CD $8+\mathrm{T}$ cell exhaustion are associated with progression of COVID-19. Infect Dis Poverty 9:161. https://doi.org/10.1186/s40249-020-00780-6

10. Zumla A, Hui DS, Azhar EI et al (2020) Reducing mortality from 2019-nCoV: host-directed therapies should be an option. Lancet 395:e35-e36. https://doi.org/10.1016/S0140-6736(20) 30305-6

11. Sousa FH, Casanova V, Findlay F et al (2017) Cathelicidins display conserved direct antiviral activity towards rhinovirus. Peptides 95:76-83. https://doi.org/10.1016/j.peptides.2017.07.013

12. Barlow PG, Svoboda P, Mackellar A et al (2011) Antiviral activity and increased host defense against influenza infection elicited by the human cathelicidin LL-37. PLoS ONE 6:e25333. https://doi. org/10.1371/journal.pone.0025333

13. Tripathi S, Tecle T, Verma A et al (2013) The human cathelicidin LL-37 inhibits influenza A viruses through a mechanism distinct from that of surfactant protein D or defensins. J Gen Virol 94:40-49. https://doi.org/10.1099/vir.0.045013-0

14. Rawson ES, Miles MP, Larson-Meyer DE (2018) Dietary supplements for health, adaptation, and recovery in athletes. Int J Sport Nutr Exerc Metab 28:188-199. https://doi.org/10.1123/ijsnem. 2017-0340

15. He CS, Fraser WD, Tang J et al (2016) The effect of 14 weeks of vitamin D 3 supplementation on antimicrobial peptides and proteins in athletes. J Sports Sci 34:67-74. https://doi.org/10.1080/ 02640414.2015.1033642

16. Pastuszak-Lewandoska D, Domańska-Senderowska D, Kiszałkiewicz J et al (2020) Expression levels of selected cytokines and microRNAs in response to vitamin D supplementation in ultra-marathon runners. Eur J Sport Sci 20:219-228. https://doi.org/10.1080/17461391.2019.1635649

17. Terra R, da Silva SAG, Pinto VS et al (2012) Effect of exercise on immune system: response, adaptation and cell signaling. Revista Brasileira de Medicina do Esporte 18:208-214. https://doi.org/10. 1590/S1517-86922012000300015

18. El-kader SMA, Al-Shreef FM (2018) Inflammatory cytokines and immune system modulation by aerobic versus resisted exercise training for elderly. Afr Health Sci 18:120-131. https://doi.org/ 10.4314/ahs.v18i1.16

19. Paolucci EM, Loukov D, Bowdish DME et al (2018) Exercise reduces depression and inflammation but intensity matters. Biol Psychol 133:79-84. https://doi.org/10.1016/j.biopsycho.2018.01. 015

20. Ferreira GA, Felippe LC, Bertuzzi R et al (2018) The effects of acute and chronic sprint-interval training on cytokine responses are independent of prior caffeine intake. Front Physiol 9:671. https://doi.org/10.3389/fphys.2018.00671

21. Senna SM, Torres MK, Lopes DA et al (2016) Moderate physical training attenuates perinatal low-protein-induced spleen lymphocyte apoptosis in endotoxemic adult offspring rats. Eur J Nutr 55:1113-1122. https://doi.org/10.1007/s00394-015-0925-y

22. Bigley AB, Rezvani K, Pistillo M et al (2015) Acute exercise preferentially redeploys NK-cells with a highly differentiated phenotype and augments cytotoxicity against lymphoma and multiple myeloma target cells. Part II: impact of latent cytomegalovirus infection and catecholamine sensitivity. Brain Behav Immun 49:59-65. https://doi.org/10.1016/j.bbi.2014.12.027

23. Sellami M, Gasmi M, Denham J et al (2018) Effects of acute and chronic exercise on immunological parameters in the elderly aged: can physical activity counteract the effects of aging? Front Immunol 9:2187. https://doi.org/10.3389/fimmu.2018.02187

24. Simpson RJ, Kunz H, Agha N et al (2015) Exercise and the regulation of immune functions. Prog Mol Biol Transl Sci 135:355-379. https://doi.org/10.1016/bs.pmbts.2015.08.001

25. Leandro CG, Silva WTF, Lima-Silva AE (2020) Covid-19 and exercise-induced immunomodulation. NeuroImmunoModulation 27:75-78. https://doi.org/10.1159/000508951 
26. Jamilloux Y, Gerfaud-Valentin M, Sève P (2020) Intravenous anakinra for cytokine storm syndromes. Lancet Rheumatol 2:E521-E522. https://doi.org/10.1016/S2665-9913(20)30225-3

27. Mehta P, Porter JC, Manson JJ et al (2020) Therapeutic blockade of granulocyte macrophage colony-stimulating factor in COVID-19-associated hyperinflammation: challenges and opportunities. Lancet Respir Med 8:822-830. https://doi.org/ 10.1016/S2213-2600(20)30267-8

28. Svensson M, Lexell J, Deierborg T (2015) Effects of physical exercise on neuroinflammation, neuroplasticity, neurodegeneration, and behavior. Neurorehabil Neural Repair 29:577-589. https://doi.org/10.1177/1545968314562108

29. Steensberg A, Fischer CP, Keller C et al (2003) IL-6 enhances plasma IL-1 ra, IL-10, and cortisol in humans. Am J Physiol Metab 285:E433-E437. https://doi.org/10.1152/ajpendo.00074. 2003

30. Scheffer DL, Latini A (2020) Exercise-induced immune system response: anti-inflammatory status on peripheral and central organs. Biochim Biophys Acta Mol Basis Dis 1866:165823. https://doi.org/10.1016/j.bbadis.2020.165823

31. Fischer CP (2006) Interleukin-6 in acute exercise and training: what is the biological relevance? Exerc Immunol Rev 12:6-33

32. Wedell-Neergaard AS, Lang Lehrskov L, Christensen RH et al (2019) Exercise-induced changes in visceral adipose tissue mass are regulated by IL- 6 signaling: a randomized controlled trial. Cell Metab 29:844-855. https://doi.org/10.1016/j.cmet.2018. 12.007

33. Pedersen BK (2017) Anti-inflammatory effects of exercise: role in diabetes and cardiovascular disease. Eur J Clin Investig 47:600611. https://doi.org/10.1111/eci.12781

34. Kamen DL, Tangpricha V (2010) Vitamin D and molecular actions on the immune system: modulation of innate and autoimmunity. J Mol Med 88:441-450. https://doi.org/10.1007/ s00109-010-0590-9

35. White JH (2012) Vitamin D metabolism and signaling in the immune system. Rev Endocr Metab Disord 13:21-29. https://doi. org/10.1007/s11154-011-9195-z

36. Martineau AR, Jolliffe DA, Hooper RL et al (2017) Vitamin D supplementation to prevent acute respiratory tract infections: systematic review and meta-analysis of individual participant data. BMJ 356:i6583. https://doi.org/10.1136/bmj.i6583

37. Gonçalves TJM, Gonçalves SEAB, Guarnieri A et al (2020) Prevalence of obesity and hypovitaminosis D in elderly with severe acute respiratory syndrome coronavirus 2 (SARS-CoV-2). Clin Nutr ESPEN 40:110-114. https://doi.org/10.1016/j.clnesp.2020. 10.008

38. Laird E, Rhodes J, Kenny RA (2020) Vitamin D and inflammation: potential implications for severity of Covid-19. Ir Med J 113:81

39. Merzon E, Tworowski D, Gorohovski A et al (2020) Low plasma $25(\mathrm{OH})$ vitamin D level is associated with increased risk of COVID-19 infection: an Israeli population-based study. FEBS J 287:3693-3702. https://doi.org/10.1111/febs.15495

40. Liu PT, Stenger S, Li H et al (2006) Toll-like receptor triggering of a vitamin D-mediated human antimicrobial response. Science 311:1773-1783. https://doi.org/10.1126/science.1123933

41. Xu J, Yang J, Chen J et al (2017) Vitamin D alleviates lipopolysaccharide-induced acute lung injury via regulation of the reninangiotensin system. Mol Med Rep 2017:7432-7438. https://doi. org/10.3892/mmr.2017.7546

42. Bae M, Kim H (2020) The role of Vitamin C, Vitamin D, and selenium in immune system against COVID-19. Molecules 25:5346. https://doi.org/10.3390/moléculas25225346

43. Campbell PA, Young MW, Lee RC (2020) Vitamin D clinical pharmacology: relevance to COVID-19 pathogenesis. J Natl Med Assoc. https://doi.org/10.1016/j.jnma.2020.09.152
44. Mora RJ, Iwata M, von Andrian UH (2008) Vitamin effects on the immune system: vitamins A and D take centre stage. Nat Rev Immunol 8:685-698. https://doi.org/10.1038/nri2378

45. Prietl B, Treiber G, Pieber T et al (2013) Vitamin D and immune function. Nutrients 5:2502-2521. https://doi.org/10.3390/nu507 2502

46. Kumar R, Rathi H, Haq A et al (2021) Putative roles of vitamin $\mathrm{D}$ in modulating immune response and immunopathology associated with COVID-19. Virus Res 292:198235. https://doi.org/ 10.1016/j.virusres.2020.198235

47. Cantorna MT, Snyder L, Lin YD et al (2015) Vitamin D and 1,25(OH)2D regulation of $\mathrm{T}$ cells. Nutrients 7:3011-3021. https://doi.org/10.3390/nu7043011

48. Chatterjee S, Sengupta T, Majumder S et al (2020) COVID19: a probable role of the anticoagulant Protein $S$ in managing COVID-19-associated coagulopathy. Aging 12:15954-15961. https://doi.org/10.18632/aging.103869

49. Ali MAM, Spinler AS (2021) COVID-19 and thrombosis: from bench to bedside. Trends Cardiovasc Med 31:143-160. https:// doi.org/10.1016/j.tcm.2020.12.004

50. Sengupta T, Majumder R, Majumder S (2021) Role of vitamin $\mathrm{D}$ in treating COVID-19-associated coagulopathy: problems and perspectives. Mol Cell Biochem 476:2421-2427. https://doi. org/10.1007/s11010-021-04093-6

51. Meehan M, Penckofer S (2014) The role of vitamin D in the aging adult. J Aging Gerontol 2:60-71. https://doi.org/10. 12974/2309-6128.2014.02.02.1

52. Abraham J, Dowling K, Florentine S (2021) Can optimum solar radiation exposure or supplemented vitamin $\mathrm{D}$ intake reduce the severity of COVID-19 symptoms? Int J Environ Res Public Health 18:740. https://doi.org/10.3390/ijerph18020740

53. Bilezikian JP, Bikle D, Hewison M et al (2020) Mechanisms in endocrinology: vitamin D and COVID-19. Eur J Endocrinol 183:R133-R147. https://doi.org/10.1530/EJE-20-0665

54. Machado PRL, Araújo MLAB, Carvalho L et al (2004) Mecanismos de resposta imune às infecções. An Bras Dermatol 79:647-662. https://doi.org/10.1590/S0365-059620040006000 02

55. Aranow C (2011) Vitamin D and the immune system. J Investig Med 59:881-886. https://doi.org/10.2310/JIM.0b013e3182 $1 \mathrm{~b} 8755$

56. Adams JS, Ren S, Liu PT et al (2009) Vitamin D-directed rheostatic regulation of monocyte antibacterial responses. J Immunol 182:4289-4295. https://doi.org/10.4049/jimmunol.0803736

57. Charoenngam N, Shirvani A, Holick MF (2021) Vitamin D and Its Potential Benefit for the COVID-19 Pandemic. Endocr Pract 27:484-493. https://doi.org/10.1016/j.eprac.2021.03.006

58. Uberti F, Lattuada D, Morsanuto V et al (2014) Vitamin D protects human endothelial cells from oxidative stress through the autophagic and survival pathways. J Clin Endocrinol Metab 99:1367-1374. https://doi.org/10.1210/jc.2013-2103

59. Todd JJ, McSorley EM, Madigan SM et al (2017) Oral spray wintertime vitamin D 3 supplementation has no impact on inflammation in Gaelic footballers. Scand J Med Sci Sports 27:1300-1307. https://doi.org/10.1111/sms.12785

60. Banchereau J, Steinman RM (1998) Dendritic cells and the control of immunity. Nature 392:245-252. https://doi.org/10.1038/32588

61. Dickie LJ, Church LD, Coulthard LR et al (2010) Vitamin D3 down-regulates intracellular Toll-like receptor 9 expression and Toll-like receptor 9-induced IL-6 production in human monocytes. Rheumatology 49:1466-1471. https://doi.org/10.1093/ rheumatology/keq124

62. Jevalikar G, Mithal A, Singh A et al (2021) Lack of association of baseline 25-hydroxyvitamin D levels with disease severity and mortality in Indian patients hospitalized for COVID-19. Sci Rep 11:6258. https://doi.org/10.1038/s41598-021-85809-y 
63. Güven M, Gültekin H (2021) The effect of high-dose parenteral vitamin D3 on COVID-19-related inhospital mortality in critical COVID-19 patients during intensive care unit admission: an observational cohort study. Eur J Clin Nutr. https://doi.org/10. 1038/s41430-021-00984-5

64. Jain A, Chaurasia R, Sengar NS et al (2020) Analysis of vitamin $\mathrm{D}$ level among asymptomatic and critically ill COVID-19 patients and its correlation with inflammatory markers. Sci Rep 10:20191. https://doi.org/10.1038/s41598-020-77093-z

65. Bobart LF, Dietrich MA, Johannsen N et al (2017) Emerging relationships between vitamin D status, physical activity habits, and immune indices in college-aged females. Int J Vitam Nutr Res 87:49-58. https://doi.org/10.1024/0300-9831/a000424

66. Mercola J, Grant WB, Wagner CL (2020) Evidence regarding vitamin D and risk of COVID-19 and its severity. Nutrients 12:3361. https://doi.org/10.3390/nu12113361
67. Grant WB, Lahore H, Rockwell MS (2020) The benefits of vitamin D supplementation for athletes: better performance and reduced risk of COVID-19. Nutrients 12:3741. https://doi.org/ 10.3390/nu12123741

68. Yang YC, Chou CL, Kao CL (2020) Exercise, nutrition, and medication considerations in the light of the COVID pandemic, with specific focus on geriatric population: a literature review. J Chin Med Assoc 83:977-980. https://doi.org/10.1097/JCMA.00000 00000000393

Publisher's Note Springer Nature remains neutral with regard to jurisdictional claims in published maps and institutional affiliations. 